\title{
Semi-automated Tip Snip cloning of restriction fragments into and out of plasmid polylinkers
}

\author{
Ichiro Matsumura \\ Emory University School of Medicine, Department of Biochemistry, O. Wayne Rollins Research Center, Atlanta, GA
}

BioTechniques 62:99-106 (March 2017) doi 10.2144/000114522

Keywords: molecular cloning; laboratory automation; synthetic biology; restriction endonuclease; genetic engineering

Supplementary material for this article is available at www.BioTechniques.com/article/114522.

Synthetic biologists rely on semi-synthetic recombinant plasmids, but DNA synthesis is constrained by practical limits on length, accuracy, and sequence composition. Cloned DNA parts can be assembled into longer constructs via subcloning, but conventional methods are labor-intensive. One-pot recombination reactions are more convenient but harder to troubleshoot, and those that depend on PCR to create fragments with compatible ends necessitate re-sequencing. The Tip Snip protocol described here enables the subcloning of an insert from one plasmid polylinker into another without PCR or gel purification steps. Cohesive ends of unwanted restriction fragments are snipped off by additional restriction endonucleases. The resulting short fragments (snippets) are eliminated by hybridization to complementary oligonucleotides (anti-snippets) and subsequent size-selection spin-column chromatography. Unwanted linear donor vectors are ligated to double-stranded oligonucleotides (unlinkers) so that only the desired insert and recipient plasmid form circular DNA capable of transforming bacteria. This new method is compatible with high-throughput processing and automated liquid handling, and because no specialized vectors, reagents, selection schemes, or analytical techniques are required, the barriers to adoption are low.

DNA synthesis costs are decreasing (1), but the assembly and cloning of synthetic DNA remains relatively labor-intensive and expensive. Nucleoside phosphoramidites are chemically synthesized on large scales. Single-stranded oligonucleotides and double-stranded synthetic genes are custom manufactured by machines, so turnover is rapid, throughput is high, and production costs are relatively low. Automation and miniaturization have decreased the per-unit cost of synthesizing gene-length ( $\leq 2 \mathrm{~kb})$ DNAs, but further innovations are required to overcome practical limitations in length, nucleotide composition, accuracy, and yield (1). It is not yet feasible to have every new construct synthesized with its vector de novo, so PCR products and synthetic genes are most often cloned into plasmids and then sequenced.

Cloned parts are often assembled into larger constructs by subcloning, but this classical approach is recalcitrant to automation for three reasons. First, robots that can load agarose gels and purify particular restriction fragments have not yet been invented. Furthermore, DNA purification, restriction digests, and ligation reactions aren't reliably efficient, making monitoring and troubleshooting necessary. Finally, the design of cloning experiments is idiosyncratic, so the development of software algorithms that emulate decision making by experienced molecular biologists is non-trivial. The per-unit labor cost of subcloning ( $\sim 10 \mathrm{~h}$ of labor per attempt, not including incubation times) far exceeds those of reagents (e.g., enzymes and purification kits).

The high cost of molecular cloning has motivated the invention of new methods (2-5). In general, one-pot sequence-specific recombination reactions, such as those catalyzed by recombinases (e.g., Gateway cloning) (6, 7), thermostable polymerases (overlap extension PCR) (8), thermostable ligases (ligase chain reaction) (9), or combinations of exonuclease, polymerase, and ligase (Gibson assembly or ligase-independent cloning $(10,11)$ are the most amenable to high-throughput and automated techniques (12). These protocols are less labor-intensive than traditional cloning workflows with discrete steps but are more difficult to troubleshoot. Another drawback of many seamless assembly techniques is their reliance on PCR or gene synthesis to create fragments with compatible ends. Every part must be re-sequenced each time it is seamlessly combined with another element and re-cloned (Special News Report. Weaver, J. 2015. BioTechniques. 59:II-III.), because the DNA polymerase I homologs used in PCR are three to five orders of magnitude less accurate than those responsible for in vivo plasmid replication and repair. Next-generation sequencing techniques lower per-unit cost, but they cannot be applied to individual plasmids. Thus, many synthetic biologists continue to assemble parts by manual subcloning.

\section{METHOD SUMMARY}

Tip Snip cloning uses restriction enzymes to shorten unwanted DNA fragments; the unwanted sticky ends are then neutralized by synthetic oligonucleotides. By eliminating the need to gel purify the desired restriction fragments, Tip Snip enables automation of the entire subcloning workflow. 
I therefore sought a way to automate the conventional restriction endonuclease/T4 DNA ligase-dependent subcloning workflow. Golden Gate assembly, which utilizes type IIS restriction endonucleases (13), and 2ab assembly (14), which utilizes plasmids with two selectable markers separated by a unique restriction site, obviate gel purification but necessitate the employment of specialized vectors incompatible with those of other cloning standards. The three antibiotic assembly (3A) protocol (15) was specifically designed to assemble parts compatible with the seminal BioBrick Assembly Standard (RFC10) used by many synthetic biologists (16). Two donor plasmids carrying parts and a recipient plasmid encoding a counter-selection marker along with a selectable marker different than those of the donors are digested with different pairs of restriction enzymes. All six of the resulting restriction fragments are ligated together and used to transform Escherichia coli. The desired recombinant construct is distinguished from the parental plasmids using an antibiotic and the counter-selection scheme. This technique circumvents gel purification, but sacrifices efficiency for convenience. Three-fragment ligations don't occur as frequently as two-fragment reactions, particularly when three other unwanted fragments with compatible cohesive ends are present. The extraneous DNA also inhibits heat shock transformation of chemically competent $E$. coli (17), and electroporation is sensitive to salts in ligation reactions so it is less amenable to high-throughput experiments. Here, I describe a set of expedients that in combination facilitate efficient and reliable cloning of DNA into or out of almost any existing plasmid polylinker (multiple cloning site) without the need for PCR amplification or gel purification.

\section{Materials and methods}

The approach described here builds upon the following classical cloning techniques (18), except as noted. A more detailed step-by-step protocol is included in the Supplementary Material. Plasmids were purified from transformed E. coli using silica spin columns (QIAGEN, Valencia, CA) and hydrated Sephadex G-50 (GE Healthcare Life Sciences, Pittsburgh, PA) in empty spin columns (Epoch Life Science, Missouri City, TX) as directed by their manufacturers. Restriction digests were set up as recommended by the supplier [New England BioLabs (NEB), Ipswich, MA]. Whenever possible $2 \mu \mathrm{g}$ of DNA (6 nM for a $5 \mathrm{~kb}$ plasmid) were digested to completion (or nearly so) at $37^{\circ} \mathrm{C}$ overnight with $20-40$ $\mathrm{U}$ of each restriction enzyme (1-2 nM) in $100 \mu \mathrm{L}$ total reaction volume. Approximately $20 \mathrm{fmol}$ of digested, purified recipient plasmid and 20 fmol of digested insert (and donor plasmid) were reacted with 0.3-1 Weiss units of T4 DNA ligase in NEB T4 DNA ligase buffer containing $5 \%$ polyethylene glycol (molecular weight: 8000) (19). The reactions were temperature cycled in a Bio-Rad (Hercules, CA) MJ mini thermocycler between $10^{\circ} \mathrm{C}$ for $30 \mathrm{~s}$ and $30^{\circ} \mathrm{C}$ for 30 s for a total of $4-18 \mathrm{~h}$ (20).

Table 1. Anti-snippet oligonucleotide sequences.

\begin{tabular}{|c|c|c|}
\hline Name & Snippet fragment & Anti-snippet (5' to $3^{\prime}$ ) \\
\hline $\begin{array}{l}\text { BioBrick prefix } \\
\text { EcoRI-Xbal }\end{array}$ & $\begin{array}{l}5^{\prime} \text {-AATTCGCGGCCGCTT } \\
\text { gcgccggcgaagatc-5. }\end{array}$ & aagcggccgcgaattG \\
\hline $\begin{array}{l}\text { BioBrick suffix } \\
\text { Spel-PstI }\end{array}$ & $\begin{array}{c}5^{\prime}-\text { CTAGTAGCGGCCGCTGCA }^{\prime} \\
\text { atcgceggcg-5 }\end{array}$ & $\begin{array}{l}\text { ctgcagcggccgctac- } \\
\text { tagt }\end{array}$ \\
\hline $\begin{array}{l}\text { External* } \\
\text { Kpnl-EcoRI }\end{array}$ & $\begin{array}{l}5^{\prime} \text {-CTTAATTAAGACGTCGG } \\
\text { catggaattaattctgcagccttaa-5 }\end{array}$ & $\begin{array}{l}\text { attccgacgtcttaat- } \\
\text { taaggta }\end{array}$ \\
\hline $\begin{array}{l}\text { External* } \\
\text { Pstl-Sacl }\end{array}$ & $\begin{array}{l}5^{\prime}-\text { GCATCGATGTTTAAACGAGCT } \\
\text { acgtcgtagctacaaatttgc-5' }\end{array}$ & $\begin{array}{l}\text { TGCAGCATCGATGTT- } \\
\text { TAAACGAG }\end{array}$ \\
\hline
\end{tabular}

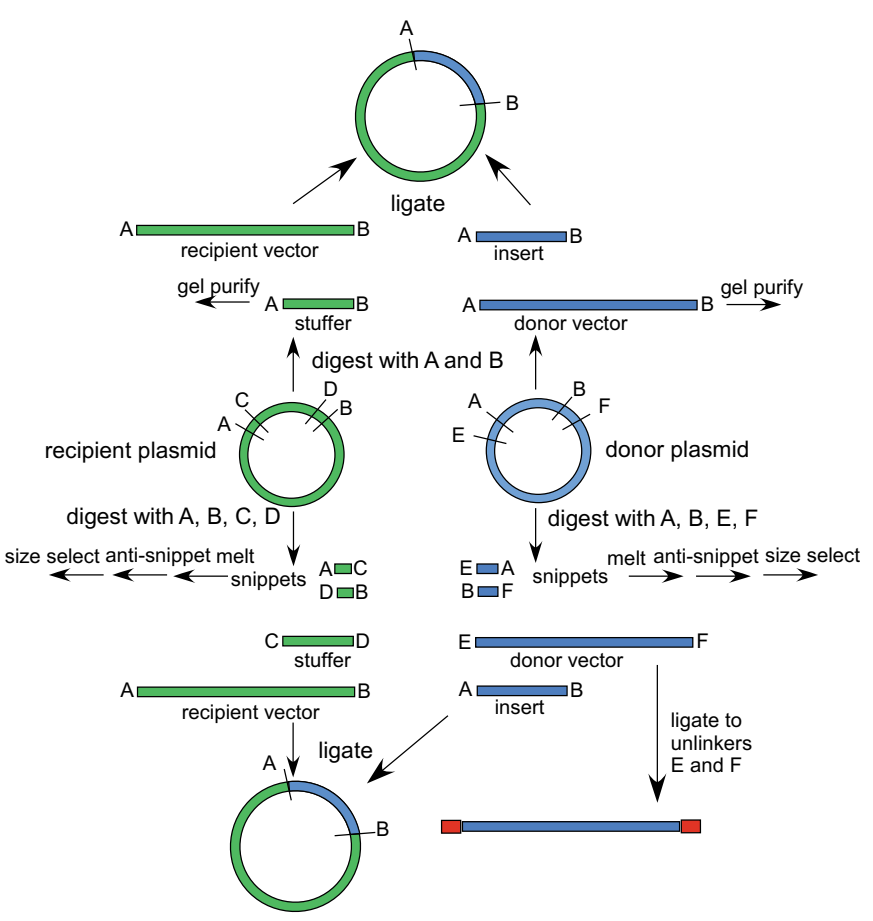

Figure 1. Conventional (middle to top) and Tip Snip (middle to bottom) subcloning workflows. Conventional digestion of donor and recipient plasmids with two restriction endonucleases each ( $A$ and $B$ ) creates four fragments: insert, unwanted donor plasmid, recipient plasmid and unwanted stuffer fragment. The unwanted fragments are traditionally eliminated manually via agarose gel electrophoresis and subsequent gel extraction of the desired bands. The Tip Snip strategy enables the automated subcloning of an insert from one polylinker (multiple cloning site) to another. Unwanted fragments are shortened slightly by additional restriction endonucleases ( $C$ and $\mathrm{D}$ or $\mathrm{E}$ and F). The unwanted short fragments (snippets) are heat denatured, hybridized to complementary oligonucleotides (anti-snippets), and eliminated via automated size-selection chromatography. The cohesive ends of the unwanted donor plasmid are made blunt by ligating them to oligonucleotide pairs that form cohesive ends (unlinkers). Only the recipient vector and insert remain intact to form circular plasmids capable of transforming Escherichia coli.

Chemically competent E. coli OmniMax2 cells (Thermo Fisher Scientific, Waltham, MA) were prepared according to Inoue et al. (17). For each transformation, up to $1.25 \mathrm{ng}$ total DNA in ligation reactions were used to transform $25 \mu \mathrm{L}$ of competent cells in the thermocycler. The transformants were spread on lysogeny broth medium (LB) agar plates containing $100 \mu \mathrm{g} / \mathrm{mL}$ ampicillin. Some agar plates also contained inducer and a histochemical substrate as described below. Some colonies were adsorbed to a nitrocellulose filter and transferred colony-side up to fresh LB-ampicillin plates supplemented with inducer (1 mM IPTG, 10 mg/mL tetracycline, $0.4 \%$ L-arabinose or $0.4 \%$ rhamnose) and $2 \mathrm{mg} / 25 \mathrm{~mL}$ plate X-gal (for colonies carrying lac $Z$ expression vectors). Additional information the reagents and materials used to culture the bacteria can be found in the Supplementary Material.

\section{Results and discussion}

The Tip Snip cloning workflow begins with plasmids prepared via alkaline lysis and silica spin-column chromatography. A QIAcube robotic workstation (QIAGEN) can be used to automate this and other purification protocols. To eliminate small molecules that might inhibit restriction endonucleases or broaden their sequence specificity (21), plasmids are further purified via manual gel-filtration spin-column chromatography. Recipient and donor plasmids are digested as usual 

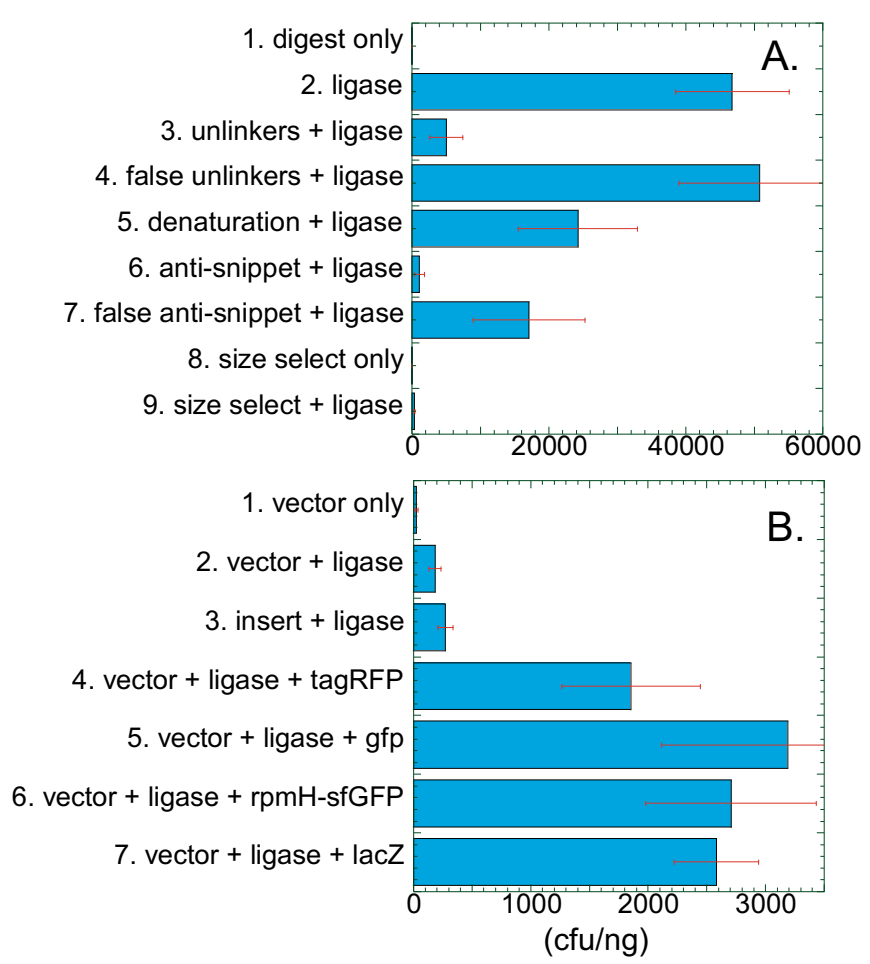

Figure 2. Tip Snip cloning techniques demonstrated via ligation and bacterial transformation. (A) Cloning plasmid IMBB2.4-pUC57-mini (Supplementary Material) was digested with four different pairs of restriction endonucleases, thereby releasing small restriction fragments (Column 1 from the top). The digestion products were reacted with T4 DNA ligase in the presence of buffer (Column 2), and unlinker (Column 3), or unlinker with overhangs that did not match those of the restriction fragments (Column 4). Alternatively, the digestion products were heat-denatured and cooled in the presence of buffer (Column 5), anti-snippets (Column 6), or anti-snippets that failed to complement either strand of the small restriction fragment (Column 7) and then reacted with T4 DNA ligase. Finally, the digestion products were purified via the QIAGEN GeneRead Size Selection protocol. The purified fragments were reacted with either buffer (Column 8) or T4 DNA ligase (Column 9). All samples were used to transform Escherichia coli cells, which were spread on lysogeny broth (LB) agar plates supplemented with ampicillin. The numbers represent average colony counts from four experiments, each of which employed a different pair of restriction endonucleases. (B) Four recipient plasmids, each encoding a different promoter, were digested with Spel and Pstl, thereby releasing short restriction fragments. Four donor plasmids, each encoding a different reporter enzyme, were digested with Xbal, Pstl, and $E c o R I$, thereby releasing insert and a short restriction fragment. The digestion products were heat denatured and cooled in the presence of a complementary oligonucleotide (anti-snippet), then purified via the QIAGEN GeneRead Size Selection protocol. The recipient plasmid (Column 2), the insert (and donor plasmid, Column 3) or combinations of recipient and insert (Columns 4-7) were reacted with T4 DNA ligase. Each reaction, as well as the unligated recipient plasmid (Column 1), were separately used to transform $E$. coli. The numbers represent average colony counts from four experiments, each employing a different recipient plasmid.

Table 2. Unlinker oligonucleotide pair sequences.

\begin{tabular}{|c|c|c|}
\hline Restriction site & Overhangs & Unlinker pair \\
\hline EcoRI & $\begin{array}{l}5^{\prime}-\mathrm{G} \text { AATTC-3' } \\
3^{\prime} \text {-CTTAA G-5' }\end{array}$ & $\begin{array}{r}5^{\prime} \text {-AATTaacgccetgaaggcgatt-3 } \\
\text { ttgcgggacttccgctaa-5 }\end{array}$ \\
\hline Pstl & $\begin{array}{l}5^{\prime}-\mathrm{CTGCA}^{\prime} \text { G-3' } \\
3^{\prime}-\mathrm{G} \quad \text { ACGTC-5 }\end{array}$ & $\begin{array}{l}5^{\prime} \text {-gcagcaggtggacgtgTGCA-3' } \\
3^{\prime} \text { - cgtcgtccacctgcac }\end{array}$ \\
\hline Kpn!* & $\begin{array}{l}5^{\prime} \text {-GGTAC } \mathrm{C}^{\prime} 3^{\prime} \\
3^{\prime}-\mathrm{C} \quad \text { CATGG-5' }\end{array}$ & $\begin{array}{l}5^{\prime} \text {-cgagatgcccaagcagcGTAC-3' } \\
3^{\prime} \text {-gctctacgggttcgtcg }\end{array}$ \\
\hline Sacl* & $\begin{array}{l}5^{\prime}-\text { GAGCT } \\
3^{\prime}-\mathrm{C}-3^{\prime}\end{array}$ & $\begin{array}{l}5^{\prime} \text {-agctcaccgcggtgctAGCT-3' } \\
3^{\prime} \text {-tcgagtggcgccacga }\end{array}$ \\
\hline
\end{tabular}

*External unlinkers that ligate to donor plasmids digested with Kpnl or Pstl are specific for IMBB2.4 and were not used in this study. with restriction enzymes that produce fragments with compatible cohesive ends. Additional restriction enzymes recognizing sites in the polylinker are used to shorten undesired restriction fragments (the stuffer fragment of the recipient plasmid or the donor plasmid of a subcloning experiment) (Figure 1).

The digests are heated to denature the short restriction fragments (called snippets) and then cooled to anneal one strand to a molar excess of a synthetic oligonucleotide (anti-snippet), thereby displacing one strand of the original fragment and forming short double-stranded hybrid DNAs with ends that cannot anneal to those of the desired vector or insert fragments (Table 1). All short single- and doublestranded DNAs are then eliminated using the QIAGEN GeneRead Size Selection Kit; a custom protocol for the QIAcube automates the addition of anti-snippets, denaturation, annealing, and silica spincolumn chromatography (Supplementary Material). Purified mixtures of restriction fragments are combined and reacted with T4 DNA ligase in a thermocycler (20). Inclusion of oligonucleotide pairs that hybridize to form cohesive ends compatible with those of unwanted donor plasmids (unlinkers) (Table 2) prevent the re-circularization of those fragments (Figure 1). Ligation reactions are used to transform chemically competent $E$. coli, and recombinant plasmids are confirmed via plasmid mini-preps and restriction mapping.

The Tip Snip strategy is intentionally redundant in several ways. Purification of plasmids via silica and size-exclusion chromatography diminishes the probability that subsequent restriction digests will be incomplete. Snippets are neutralized with anti-snippets and eliminated via size-selection spin-column chromatography. Unlinkers are used to prevent multiply digested donor plasmid fragments from ligating to other copies of themselves; such ligations produce long inverted repeats, which are not usually viable in vivo unless some further rearrangement occurs (22). These procedural redundancies could be eliminated at the risk of increasing costs associated with screening clones, troubleshooting, and repeating experiments.

The utility of size-exclusion chromatography for the elimination of restriction endonuclease inhibitors, size-selection chromatography for the elimination of small DNA fragments, and unlinkers for the inhibition of ligation reactions was initially demonstrated in agarose gels (Supplementary Figure S1). Unlinkers, size selection, and antisnippets were also characterized by digesting plasmids, re-ligating them under various conditions, and transforming $E$. coli as follows: Cloning plasmid IMBB2.4-pUC57-mini was digested with pairs of restriction endonucleases (EcoRl/Xbal, Spel/Pstl, Kpnl/EcoRl or $P s t / / S a c l)$ so that snippets were released. Aliquots of each digest were mixed with a molar excess of compatible unlinker (or water or mismatched unlinker). Other aliquots were combined with the corresponding anti-snippets (or water or mismatched anti-snippets), incubated for $30 \mathrm{~s}$ at $70^{\circ} \mathrm{C}$, and cooled slowly to room temperature. One additional aliquot of each digest was purified using the QIAGEN GeneRead Size Selection protocol. All aliquots were reacted with T4 DNA ligase, diluted, heat killed, and used to transform E. coliOmniMax2 cells, which were spread on LB plates supplemented with ampicillin. The sequence-matched unlinker, anti-snippets, and size selection reduced the re-ligation frequency of the plasmid to its snippet insert by 92\%, 99\%, and 99\%, respectively, compared with the corresponding untreated control (Figure 2A). Mismatched unlinker and anti-snippets exhibited no inhibitory effects, demonstrating that their activities are sequence-dependent as expected.

A series of reporter gene expression vectors were constructed using only the four BioBrick restriction endonucleases, namely EcoRl, 
Xbal, Spel, and Pstl, to demonstrate compatibility with this widely used cloning standard (Supplementary Figure S1). Four BioBrickcompatible variants of promoter regions, tacl (23), tac-tetO (identical to tacl but with a tet instead of lac operator), $\operatorname{araBAD}(24)$, and rhaBAD (25) were synthesized (see Supplementary Material for sequences) and digested overnight with restriction endonucleases EcoRI and Pstl. The QIAquick PCR purification protocol was used to purify the large restriction fragments. Cloning vector IMBB2.4-pUC57-mini was digested with EcoRI, Pstl, Xbal and Spel. A custom QIAcube protocol (Supplementary Material) then added anti-snippets that partially complement the BioBrick prefix and suffix. The reaction was heated to $70^{\circ} \mathrm{C}$ to denature the snippets and then cooled slowly to room temperature.

The large fragments were purified with the GeneRead Size Selection protocol as directed by the manufacturer (QIAGEN), albeit with the custom QIAcube protocol. The concentrations of purified plasmid and synthetic DNA fragments were measured with a BioTek Take3 plate in a Synergy 2 microtiter plate spectrophotometer. The fragments were then ligated together in reactions with T4 DNA ligase. The ligation reactions were used to transform E. coli strain OmniMax2 and the cells were spread on LB agar plates supplemented with ampicillin. The number of colonies on plates corresponding to vector + insert + ligase reactions (1450 $\pm 159 \mathrm{cfu} / \mathrm{ng})$ exceeded those on the vector + ligase control plates (156 cfu/ng) by nearly an order of magnitude. The number of background colonies would have been lower if the ligation products had been digested with a restriction endonuclease that recognized an internal site within the polylinker (namely Sphl, Apal, Ndel, Bg/ll, Ncol, Sfil, EcoRV, Hindlll, BamHl or Xhol) $(26,27)$; post-ligation digestion was eschewed to show that the approach works with any BioBrick accepting vector.

Tip Snip subcloning is particularly useful for the assembly of DNA constructs because fragments don't need to be re-amplified or re-sequenced after they are recombined with others. Four BioBrick-compatible ribosome binding sites and reporter genes, those encoding tagRFP, GFP, rpmH-sfBFP, and $\beta$-galactosidase, were previously synthesized or PCR amplified (lacZ) and cloned via classical techniques into pUC-based plasmids that confer upon host cells resistance to ampicillin (Supplementary Material). The reporter genes were excised from donor plasmids by overnight digestion with Xbal, Pstl and EcoRI (Supplementary Figure S2). The recipient plasmids, which encode promoters (see above), were digested overnight with Spel and Pstl.

The custom QIAcube protocol was used to add anti-snippets complementary to the small restriction fragments (BioBrick prefix and suffix), incubate the mixture at $70^{\circ} \mathrm{C}$, cool it slowly to room temperature, and then purify the larger fragments with the GeneRead Size Selection protocol. The purified restriction fragments were analyzed by agarose gel electrophoresis. Each of the four cloned promoters was ligated to each of the four reporter genes. These ligation reactions, including controls (vector only, vector plus ligase, insert plus ligase), were incubated at $65^{\circ} \mathrm{C}$ for 10 minutes to inactivate the T4 DNA ligase and used to transform E. coli OmniMax2 cells. The transformed bacteria were spread on LB agar plates supplemented with ampicillin and incubated at $37^{\circ} \mathrm{C}$ overnight. The number of colonies on the plates corresponding to the complete ligation reactions (vector plus insert plus ligase) exceeded those on control plates (vector plus ligase or insert plus ligase) by nearly an order of magnitude (Figure 2B).

The transformed colonies were filter-lifted onto LB agar plates supplemented with inducer (IPTG, tetracycline, L-arabinose, or
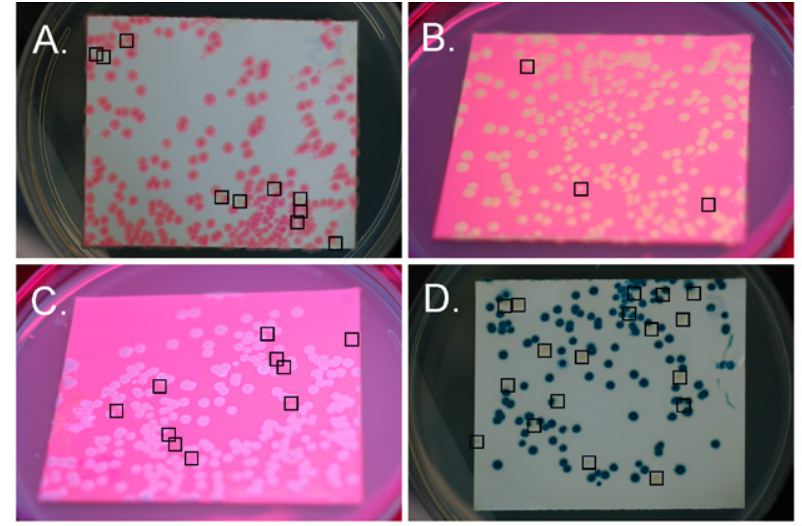

Figure 3. Filter-lifts of transformed colonies illustrate the high frequency of desired recombinant constructs. Colonies putatively transformed with recombinant plasmids - (A) araBAD-tagRFP, (B) Ptac-tetO-gfp, (C) rhaS-rhaB-rpmHsfBFP, (D) Ptac-lacO-12his-lacZ - were adsorbed to nitrocellulose filters and transferred colony-side up to fresh lysogeny broth (LB) agar plates containing ampicillin, an appropriate inducer-(A) L-arabinose, (B) tetracycline, (C) L-rhamnose, (D) IPTG) — and X-gal (for clones expected to express lacZ). The transferred colonies were induced for $16 \mathrm{~h}$ and photographed under white (tagRFP, lacZ/X-gal) or $360 \mathrm{~nm}$ light (gfp, sfBFP). Uncolored colonies are located with squares. Colony counts from all filters are listed in Supplementary Table S1.

L-rahmnose) and the histochemical marker X-gal for cells harboring plasmid-borne lac $Z$ genes. Colonies were incubated overnight at $37^{\circ} \mathrm{C}$ then photographed under white (tagRFP, lacZ) or $360 \mathrm{~nm}$ (gfp, sfBFP) light (Figure 3). The majority of filter-bound colonies that putatively carried tac-tagRFP, tac-tetO-tagRFP, araBAD-tagRFP, or rhaBAD-tagRFP turned red, while those containing tac-rpmH-sfBFP, tac-tetO-rpmH-sfBFP, araBAD-rpmH-sfBFP, or rhaBAD-rpmHsfBFP turned blue. These results confirm that all four promoters can drive downstream open reading frames and that the tagRFP and rpmH-sfBFP reporters can produce detectable signals in E. coli colonies. Most colonies that putatively carried tac-lacZ or araBADlacZ cassettes turned blue on plates containing inducer and X-gal, but those that carried tac-tetO-lacZ or rhaBAD-lacZ did not. Similarly, most colonies that putatively carried tac-tetO-gfp turned green, but those that carried tac-gfp, araBAD-gfp, and rhaBAD-gfp did not.

Overall, 11 of the 16 promoter-reporter gene combinations that were assembled produced signals that could be visualized within individual colonies (Supplementary Table S1). The vast majority of colonies on those 11 filters ( $94 \% \pm 1 \%$ ) were colored, representing desired promoter-reporter gene assemblies. No color was observed in control colonies that carried the parental plasmids (promoter only or reporter gene only). Furthermore, 5 red and 12 white colonies were picked from the araBAD-tagRFP filter (and a replicate) and propagated in liquid LB-ampicillin. Plasmids were prepared from each culture and restriction mapped. All five plasmids derived from the red colonies showed restriction fragments corresponding to araBAD-tagRFP as expected, suggesting that all of the colored colonies were recombinant. Among the 12 plasmids from the white colonies, 6 contained fragments corresponding to tagRFP only, 4 encoded araBAD only, and 2 did not produce restriction fragments consistent with either parent plasmid. These results suggest that the restriction digests were incomplete and that optimization of these reactions would have decreased background. The inclusion of Sacl (or plasmid-cleaving Cas9/single-guide RNA complex) in the initial digest of the donor plasmid (Figure 1 and Supplementary Figure S2) and the subsequent addition of the appropriate anti-snippet would also have eliminated the six tagRFP-only plasmids. 


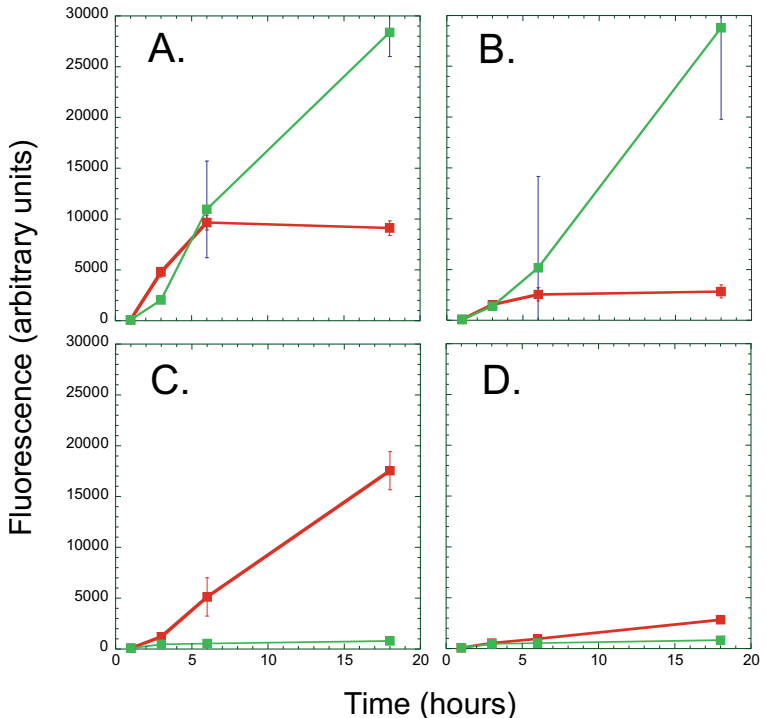

Figure 4. Induction of clones expressing tagRFP. Four individual colonies transformed with plasmids encoding (A) tac-lacO-tagRFP, (B) tac-tetOtagRFP, (C) araBAD-tagRFP, (D) rhaBAD-tagRFP, or negative controls (promoter only or tagRFP only; not shown) were propagated to stationary phase at $37^{\circ} \mathrm{C}$ in lysogeny broth (LB)-ampicillin in microtiter plates. The cultures were diluted 100-fold in duplicate and propagated to mid-log phase. Inducer-(A) IPTG, (B) tetracycline, (C) L-arabinose, (D) L-rhamnose-was added to half the micro-cultures, and fluorescence from each well (excitation 544 $\mathrm{nm}$, emission $590 \mathrm{~nm}$ ) was measured after 1, 3, 6, and $18 \mathrm{~h}$. Values are plotted in green for the uninduced cultures and in red for the induced cultures.

High-throughput subcloning enables the combinatorial assembly of variant constructs. The filter-lift experiments described above show that the different promoter-reporter gene combinations vary in efficiency. The tagRFP reporter was the most reliable of the four. Induction of the various promoter-tagRFP cassettes was measured in microtiter plate culture assays in parallel with promoter-only and tagRFP-only controls. Colonies were picked in quadruplicate and propagated at $37^{\circ} \mathrm{C}$ in LB-ampicillin micro-cultures in 96-well microtiter plates. Saturated cultures were diluted in duplicate plates containing 100-fold in fresh LB-ampicillin and propagated at $37^{\circ} \mathrm{C}$ for another $4 \mathrm{~h}$. Inducers appropriate for each promoter (IPTG, tetracycline, L-arabinose, or L-rhamnose) were added to each mid-log phase culture of one microtiter plate. Fluorescence from each well of both plates (excitation $544 \mathrm{~nm}$, emission $590 \mathrm{~nm}$ ) was measured after 1, 3, 6, and $18 \mathrm{~h}$ (Figure 4). The tac-lacO and tac-tetO promoters were strong and leaky under these conditions. Overexpression of tagRFP apparently reduced growth rates such that the signal from uninduced cells at later time points was greater from these expression vectors, on average, than that from induced cells. The araBAD and rhaBAD promoters were better repressed under non-inducing conditions, so induction led to increased signal, as expected.

Tip Snip cloning is most useful for the assembly of cloned parts into a construct without re-synthesizing or re-sequencing. It was demonstrated by combining four promoters with four reporter genes in parallel. The spin columns used in this study are also available in a 96-well format so the workflow could conceivably be scaled up to 48 $\times 48$ pairs, although an alternative to the $15 \times 100 \mathrm{~mm}$ Petri dishes used here to isolate individual clones would have to be devised. Such economies of scale could further reduce costs for investigators willing to adopt a cloning standard. It should also be possible to automate the set-up of restriction digests, ligations, and transformations so that the entire workflow, except for microbiological steps, could be executed by a liquid handler.

\section{Acknowledgments}

Brindar Sandhu tested an early version of Tip Snip cloning and found it wanting. She, Vincent Peterson, and Oskar Laur provided constructive criticism of the writing. The author was supported by NSF grants (MCB 1359575, MCB 1413062).

\section{Competing interests}

A provisional patent application disclosing the work in the article was filed by the Emory University Office of Technology Transfer with the United States Patent and Trademark Office on February 22, 2017 and assigned Serial No. 62/462,210.

\section{References}

1. Kosuri, S. and G.M. Church. 2014. Large-scale de novo DNA synthesis: technologies and applications. Nat. Methods 11:499-507.

2. Casini, A., M. Storch, G.S. Baldwin, and T. Ellis. 2015. Bricks and blueprints: methods and standards for DNA assembly. Nat. Rev. Mol. Cell Biol. 16:568-576.

3. Celie, P.H., A.H. Parret, and A. Perrakis. 2016. Recombinant cloning strategies for protein expression. Curr. Opin. Struct. Biol. 38:145-154.

4. Hartley, J.L. 2006. Cloning technologies for protein expression and purification. Curr. Opin. Biotechnol. 17:359-366.

5. Lu, Q. 2005. Seamless cloning and gene fusion. Trends Biotechnol. 23:199-207.

6. Hartley, J.L., G.F. Temple, and M.A. Brasch. 2000. DNA cloning using in vitro sitespecific recombination. Genome Res. 10:1788-1795.

7. Walhout, A.J., G.F. Temple, M.A. Brasch, J.L. Hartley, M.A. Lorson, S. van den Heuvel, and M. Vidal. 2000. GATEWAY recombinational cloning: application to the cloning of large numbers of open reading frames or ORFeomes. Methods Enzymol. 328:575-592.

8. Bryksin, A.V. and I. Matsumura. 2010. Overlap extension PCR cloning: a simple and reliable way to create recombinant plasmids. BioTechniques 48:463-465.

9. de Kok, S., L.H. Stanton, T. Slaby, M. Durot, V.F. Holmes, K.G. Patel, D. Platt, E.B. Shapland, et al. 2014. Rapid and reliable DNA assembly via ligase cycling reaction. ACS Synth. Biol. 3:97-106.

10. Gibson, D.G., L. Young, R.Y. Chuang, J.C. Venter, C.A. Hutchison 3rd, and H.O Smith. 2009. Enzymatic assembly of DNA molecules up to several hundred kilobases. Nat. Methods 6:343-345

11. Aslanidis, C. and P.J. de Jong. 1990. Ligation-independent cloning of PCR products (LIC-PCR). Nucleic Acids Res. 18:6069-6074.

12. Shih, S.C., G. Goyal, P.W. Kim, N. Koutsoubelis, J.D. Keasling, P.D. Adams, N.J. Hillson, and A.K. Singh. 2015. A Versatile Microfluidic Device for Automating Synthetic Biology. ACS Synth. Biol. 4:1151-1164.

13. Weber, E., C. Engler, R. Gruetzner, S. Werner, and S. Marillonnet. 2011. A modular cloning system for standardized assembly of multigene constructs. PLoS One 6:e16765.

14. Leguia, M., J. Brophy, D. Densmore, and J.C. Anderson. 2011. Automated assembly of standard biological parts. Methods Enzymol. 498:363-397.

15. Shetty, R., M. Lizarazo, R. Rettberg, and T.F. Knight. 2011. Assembly of BioBrick standard biological parts using three antibiotic assembly. Methods Enzymol. 498:311-326

16. Raimbault, B., J.P. Cointet, and P.B. Joly. 2016. Mapping the Emergence of Synthetic Biology. PLoS One 11:e0161522.

17. Inoue, H., H. Nojima, and H. Okayama. 1990. High efficiency transformation of Escherichia coli with plasmids. Gene 96:23-28.

18. Matsumura, I. 2015. Why Johnny can't clone: Common pitfalls and not so common solutions. BioTechniques 59:IV-XIII.

19. King, P.V. and R.W. Blakesley. 1986. Optimizing DNA Ligations for Transformation. Focus 8:1-3.

20. Lund, A.H., M. Duch, and F.S. Pedersen. 1996. Increased cloning efficiency by temperature-cycle ligation. Nucleic Acids Res. 24:800-801.

21. Robinson, C.R. and S.G. Sligar. 1993. Molecular recognition mediated by bound water. A mechanism for star activity of the restriction endonuclease EcoRI. J. Mol. Biol. 234:302-306.

22. Sambrook, S and D.W. Russell. 2001. Molecular Cloning: A Laboratory Manual, 3rd Ed, Vol. 1. Cold Spring Harbor Laboratory Press, Cold Spring Harbor, NY.

23. de Boer, H.A., L.J. Comstock, and M. Vasser. 1983. The tac promoter: a functional hybrid derived from the trp and lac promoters. Proc. Natl. Acad. Sci. USA 80:21-25.

24. Guzman, L.M., D. Belin, M.J. Carson, and J. Beckwith. 1995. Tight regulation, modulation, and high-level expression by vectors containing the arabinose PBAD promoter. J. Bacteriol. 177:4121-4130

25. Giacalone, M.J., A.M. Gentile, B.T. Lovitt, N.L. Berkley, C.W. Gunderson, and M.W. Surber. 2006. Toxic protein expression in Escherichia coli using a rhamnose-based tightly regulated and tunable promoter system. BioTechniques 40:355-364.

26. Spear, M.A. 2000. Efficient DNA subcloning through selective restriction endonuclease digestion. BioTechniques 28:660-662, 664, 666 passim.

27. Zeng, Q., M.K. Eidsness, and A.O. Summers. 1997. Near-zero background cloning of PCR products. BioTechniques 23:412-414, 416, 418.

Received 27 November 2016; accepted 05 January 2017.

Address correspondence to Ichiro Matsumura, Emory University School of Medicine, Department of Biochemistry, O. Wayne Rollins Research Center, 1510 Clifton Road NE, Room 4119 Atlanta, GA 30322. E-mail: imatsum@emory.edu

To purchase reprints of this article,contact: BioTechniques@fosterprinting.com 